\title{
Laryngeal neuroendocrine neoplasia
}

\begin{abstract}
Laryngeal neuroendocrine neoplasia is rare disease, it is the second most common laryngeal malignancy, most patients are male smoker with advanced age, supraglottic region is the most common laryngeal site, the diagnosis is depended on histopathological exam done by experienced pathologist, treatment and prognosis is determined by the type of neuroendocrine tumor
\end{abstract}

Objective: This is a little discussion in literatures about laryngeal neuroendocrine neoplasms. The objective of this study is to present a review article about this disease.
Volume 6 Issue 6 - 2017

\section{Omar Ramadan}

Independent researcher Paterson NJ, Kuwait

Correspondence: Omar Ramadan Independent researcher Paterson NJ, Kuwait, Tel 0019735639283 ,

Email omarram982@hotmail.com

Received: August 12, 2016 | Published: April 17, 2017

Keywords: laryngeal, neuroendocrine, neoplasia, carcinoid, carcinoma, tumor, submucosal, laryngectomy, smcc, paragangliomas

\section{Introduction}

Neuroendocrine tumors can occur in any part of the body, but they are mostly reported in Gastrointestinal tract and bronchial system, they are rare tumors in Head and neck area, in 1969 Goldman et al reported laryngeal neuroendocrine tumor as separate entity, and after that about 700 neuroendocrine tumor cases have been reported in English literature. Laryngeal neuroendocrine carcinoma is uncommon neoplasm accounting about $1 \%$ of laryngeal tumors, but it is the second most common laryngeal malignancy. The clinical behavior and prognosis of this neoplasia are based on the type of neuroendocrine tumor; they are divided into two divisions.

i. Epithelial type; which also can be divided into 4 types:

a. Typical carcinoid; well differentiated Tumor

b. Atypical carcinoid: moderately differentiated tumor

c. Large cell carcinoma; poorly differentiated tumor

d. Small cell carcinoma: poorly differentiated tumor

ii. Neural type: Paraganglioma. ${ }^{1-3}$

The neuroendocrine system is made up of different type of cells from central nervous system, peripheral nervous system and all other body organs, those cells have a common phenotype of producing active amines or peptides such chromogranin-A, bombesin, calcitonin, serotonin, and cholecystokinin. Neuroendocrine cells are found in larynx especially in supraglottic region. Functions of those cells are regulating local epithelial growth, oxygen sensation, affecting vasculature and autonomic nerve terminals. ${ }^{3}$

Typical carcinoid is extremely uncommon, there is only 20 cases reported in English literature, it is the least common neuroendocrine malignancy of the larynx, it is more common in male, typically patients are smoker in the sixth to eighth decade of life. A typical carcinoid neoplasia generally grows in submucosal area, and look like a polypoid or sessile mass, it is mostly seen in supraglottic area. Paraneoplastic syndrome may happen in this disease, but this condition is rare. The diagnostic workup usually include indirect laryngoscopy, which may show a submucosal polypoid mass in the supraglottic larynx, CT-Scan which is used to determine local and regional spread of the tumor.

The diagnosis is done by histopathological examination using microscopy; but it is difficult to distinguish between typical and atypical carcinoid tumor using light microscopy, so histochemical, immunohistochemical and ultrastructural examinations under the electron microscopy may be needed. The treatment of choice for typical carcinoid is total surgical resection, laryngeal conservation surgery is sufficient for small lesion, while total larygectomy is recommended for large invasive tumor. Due to unusual lymph node metastases, neck dissectopn is recommended only for clinical and pathological lymph node, the tumor is resistant to chemoradiotherapy, and prognosis is usually good. ${ }^{4-7}$

Atypical carcinoidis located mostly in the supraglottic region; most patients are male in the sixth-to-seventh decade of life. The presenting symptoms are hoarseness, dysphagia, odynophagia and dyspnea. The paraneoplastic syndrome may be associated with this tumor. The histopathological diagnosis is made by routine microscopy, but histochemical, immunohistochemical and ultrastructural examinations under the electron microscope are often needed. The differential diagnosis must basically be done against other types of neuroendocrine neoplasms, with special attention to paragangliomas. The surgical excision is the mainstay of treatment for laryngeal atypical carcinoid tumor. Depending on the site and extent of the primary tumor Partial or total laryngectomy may be indicated. As most tumors are located in supraglottic region, supraglottic laryngectomy is often the procedure of choice for supraglottic location. Elective neck dissection is indicated due to high incidence of early cervical lymph node metastasis. ${ }^{5-8}$

Large cell carcinoma LCC is a rare disease; only 17 cases were reported in English literature, it is classified as variant of atypical carcinoid, but it is also has some features of small cell carcinoma, so it may be also classified as a variant of Small Cell Carcinoma. Atypical carcinoid and Large Cell Carcinoma have quite similar histologic features, but the distinguishing mark between them is the mitotic activity which is more than 40 in LCC and less than 10 in atypical carcinoid. This neoplasia must fulfill proposed diagnostic criteria for pulmonary large cell neuroendocrine carcinoma. It is poorly differentiated tumor with more aggressive behavior than atypical carcinoid. Prognosis is poor since most patients have distant metastatic disease at the early stage and dying within 2 years. Therapy is not well standardized for this tumor, but Due to aggressive behavior of this tumor, there is a great need to develop effective systemic treatment. ${ }^{9}$

Small cell carcinoma SmCC has been seen in multiple areas of the head and neck, 160 cases has been reported in the larynx in world 
literature, it is extremely rare tumor. Definitive diagnosis is absolutely important to give an appropriate therapy to laryngeal SmCC, so other neuroendocrine neoplasms of the larynx should be clearly excluded. Histological and immunohistochemical examination by excellent pathologists are recommended to distinguish SmCC from other neuroendocrine or epithelial tumors. Since laryngeal SmCC is a lethal disease and has an extremely poor prognosis, so surgical resection is not recommended, Radiation alone did not improve survival, but it was successful in controlling the primary site of the tumor. Adjuvant chemotherapy prolongs the survival among patients. The combination of adjuvant chemotherapy and radiation therapy to local tumor site is the recommended treatment, it improves patient survival. Cyclophosphamide, doxorubicin, vincristine, methotrexate, and lomustine are the commonly used chemotherapeutic agents. Period of treatment is usually ranged from 9 to 18 month. Resistance to chemotherapy represents a poor prognostic factor. The recurrence is usually generalized and the palliative chemotherapy may be recommended for these cases. The chemotherapeutic agents commonly used should not penetrate the blood-brain barrier. Prophylactic cranial irradiation has been recommended as part of the management of this cancer. $^{5-7,10}$

Combined small cell carcinoma consists of small cell carcinoma with a component of squamous cell carcinoma or adenocarcinoma. Combined primary squamous and small cell carcinoma of the larynx is rare, and only 13 cases have been reported in English literature, it is mostly located in supraglottic region. Patients usually are male smoker with median age 55, the presenting symptom are hoarseness, dysphagia, difficulty breathing and neck lymphadenopathy. The clinical features are similar to those of small cell carcinoma. Paraneoplastic syndrome may also occur in association with laryngeal small cell carcinoma. The clinical course of combined small cell carcinoma is similar to that of small cell carcinoma of the larynx; it displays aggressive biologic behavior with early distant metastasis, the recommended treatment is combined radiotherapy with chemotherapy. Prognosis is poor with most patients were dead after 2years. ${ }^{11}$ Laryngeal paragangliomas are rare neuroendocrine tumors that arise from paraganglion cells located in submucosal layer of the larynx.

Paragangliomas are also known to appear in two forms: sporadic and familial. Multicentric forms of head and neck paragangliomas are usually a result of the familial paraganglioma syndrome. However, larynx involvement is very rare, especially in the multicentric type. It is located mostly in supraglottic area and, but a few cases reported in subglottic area. There are about 76 cases reported in world literature. Only $2 \%$ of these tumors are malignant and are often misdiagnosed as atypical carcinoids. Symptoms arise when the tumor become large enough to impair function. Supraglottic paragangliomas cause hoarseness and deglutition disorders, whereas the symptoms of subglottic tumors appear when they obstruct the airway. Familial paragangliomas and hypertension should be screened, but they are rarely associated with laryngeal paragangliomas.

MRI can detect these tumors and help to determine the vascularity of the lesion. Pentetreotide scanning can differentiate between the neuroendocrine tumors and other submucosal laryngeal lesions, making the preoperative diagnosis clearer and decreasing the need for biopsy. Immunohistochemical markers and supplementing standard histopathologic exam help to make the correct diagnosis and distinguish paragangliomas from other neuroendocrine tumors. This distinction is critical as the prognosis of paragangliomas is excellent compared to that for other neuroendocrine neoplasms. Surgery is the treatment of choice for laryngeal paraganglioma as it cure disease easily with function preservation, it is better than radiotherapy which may induce swelling, airway obstruction and destruction of cartilage. Partial conservation laryngectomy is the mainstay of treatment. Laser surgery is typically not recommended because of the vascular nature of these tumors. Preoperative angiography and embolization is not necessary for small lesion but arterial Ligation at the time of surgery is recommended. For supraglotic lesion a local excision is indicated, but there is small number of paragangliomas that may present as transventricular or infraglottic tumors, these lesions should be removed via larygofissure with or without cricoid split. Prognosis

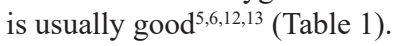

Table I Neuroendocrine Tumor Types

\begin{tabular}{|c|c|c|c|c|}
\hline & Typical Carcinoid & $\begin{array}{l}\text { Atypical } \\
\text { Carcinoid }\end{array}$ & Small Cell Carcinoma & Paraganglioma \\
\hline Reported cases & 20 & 500 & 160 & 76 \\
\hline Risk factor & Male in Sixth decade, smoker & $\begin{array}{l}\text { Male in six } \\
\text { decade, smoker }\end{array}$ & Male in sex decade, smoker & Female \\
\hline Region & Submucosal, supraglotic & $\begin{array}{l}\text { Submucosal, } \\
\text { supraglotic }\end{array}$ & $\begin{array}{l}\text { Submucusal with ulceration, } \\
\text { Supraglotc }\end{array}$ & Submucusal, Supraglotic \\
\hline Pathology & Less than 2 mitosis & 2-10 mitosis & $\begin{array}{l}\text { Over } 10 \text { mitotic activity } \\
\text { with necrosis }\end{array}$ & "Zellballen" pattern \\
\hline $\begin{array}{l}\text { Immunohistological for NSE, } \\
\text { Chromogranin, CEA, Calcitonin }\end{array}$ & Less than $2 \%$ & $2-5 \%$ & More than $20 \%$ & \\
\hline Metastasis & Rare & Common & Very Common & Rare and consider malignan \\
\hline Paraneoplastic Syndrome & + & + & ++ & + \\
\hline Treatment & Surgery & Surgery & Chemoradiotherpay & Surgery \\
\hline Prognosis & Good & Intermediate & Poor & Good \\
\hline
\end{tabular}

\section{Conclusion}

Laryngeal Neuroendocrine tumors are heterogeneous group in terms of biological behavior and pathological features. The histopatholgical diagnosis done by experienced pathologist is the most essential step in the management of these malignancies. Tumor histology type determines the management and the prognosis. They may be associated with paraneoplastic syndromes. Typical carcinoid has a good prognosis and can be managed with conservation surgery and neck dissection for lymph node metastasis. Atypical carcinoid is more aggressive and should be managed with appropriate surgical resection (partial or total laryngectomy) with neck dissection. SmCC has poor prognosis, it is usually managed with chemoradiotherapy. Paragangliom has a better prognosis, it is managed by local excision or partial laryngectomy. 


\section{Acknowledgments}

None.

\section{Conflicts of interest}

Author declartes there are no conflicts of interest.

\section{Funding}

None.

\section{References}

1. Hemalatha AL, Anoosha K, Amita K, et al. Primary Laryngeal Neuroendocrine Carcinoma-A Rare Entity with Deviant Clinical Presentation. JCDR. 2014; 8(9):FD07-FD08.

2. Chung EJ, Baek SK, Kwon SY, et al. Moderately Differentiated Neuroendocrine Carcinoma of the Larynx. Clin Exp Otorhinolaryngol. 2008;1(4):217-220.

3. Lewis JS, Ferlito A, Gnepp DR, et al. Terminology and classification of neuroendocrine neoplasms of the larynx. Laryngoscope. 2011;121(6):1187-1193.

4. Kayhan FT, Başaran EG. Typical carcinoid tumor of the larynx in a woman: a case report. J Med Case Rep. 2010;4:321.

5. Kumar LP, Armugham N, Triveni B, et al. Neuroendocrine tumor of larynx: A review of literature. Asian J Oncol. 2015;1(1):33-36.
6. Ferlito A, Silver CE, Bradford CR, et al. Neuroendocrine neoplasm of the larynx: on overview. Head Neck. 2009;31(12):1634-1646.

7. Ferlito A, Lewis JS, Rinaldo A. The evolving management of laryngeal neuroendocrine carcinomas. Eur Arch Otorhinolaryngol. 2011;268(9):1247-1248

8. Piazza C, Giudice M, Berlucchi M. A typical carcinoid tumour of the larynx treated with $\mathrm{CO} 2$ laser excision: case report. Acta Otorhinolaryngol Ital. 2003;23:43-46.

9. Lewis J, Spence DC, Chiosea S, et al. Large cell neuroendocrine carcinoma of the larynx: definition of an entity, Head Neck Pathol. 2010;4(3):198-207.

10. Susumu Nakahara, Norihiko Takemoto, Hidenori Inohara. Small Cell Carcinoma of the Larynx Treated by Concurrent Chemoradiotherapy: A Case Report. Case Reports in Otolaryngology. 2012;2012(2012):ID 316165 .

11. VR Jaiswal, MP Hoang. Primary combined squamous and small cell carcinoma of the larynx: a case report and review of the literature. Arch Pathol Lab Med. 2004;128(11):1279-1282.

12. Myssiorek D, Rinaldo A, Barnes L, et al. Laryngeal paraganglioma: an updated critical review. Acta Otolaryngol . 2004;124(9):995-999.

13. Sharifkashany S, Yazdani N, Ghazavi H, et al. Laryngeal Paraganglioma: A Case Report. Iran J Radiol. 2014;11(3):e21011. 